\title{
Src inhibition reduces NR2B surface expression and synaptic plasticity in the amygdala
}

\author{
Laleh Sinai, ${ }^{1,2,3}$ Steven Duffy, ${ }^{1}$ and John C. Roder ${ }^{1,2}$ \\ ${ }^{1}$ Mount Sinai Hospital Research Institute, Toronto, Ontario M5G 1X5, Canada; ${ }^{2}$ Institute of Medical Science, \\ Molecular and Medical Genetics, University of Toronto, Toronto, Ontario M5S 1A1, Canada
}

\begin{abstract}
The Src protein tyrosine kinase plays a central role in the regulation of $N$-methyl-D-aspartate receptor (NMDAR) activity by regulating NMDAR subunit $2 B$ (NR2B) surface expression. In the amygdala, NMDA-dependent synaptic plasticity resulting from convergent somatosensory and auditory inputs contributes to emotional memory; however, the role of Src tyrosine kinase has not been investigated. We have synthesized a Src-derived peptide, Tat-Src (40-58), that crosses the blood-brain barrier following injection and accumulates intracellularly. Tat-Src (40-58) blocks the interaction of Src with NMDA receptors. Following injection, mice demonstrate impaired amygdala-dependent cued fear conditioning, as well as impairments in an amygdala-dependent nonassociative social recognition task. The Src inhibitor decreased NR2B phosphorylation in amygdala tissue and reduced NR2B surface expression in cultured amygdala neurons with a concomitant reduction in NMDA multimer-containing dendritic puncta. In addition, preincubation of this inhibitory peptide blocked amygdalar long-term potentiation in the lateral to basolateral pathway in vitro. These results indicate that Src is a key regulator of NMDAR trafficking in the amygdala. Furthermore, Src-dependent phosphorylation of NR2B supports amygdala plasticity and amygdalar-dependent learning.
\end{abstract}

Src is a nonreceptor protein tyrosine kinase that is expressed widely throughout the central nervous system. Src plays an important role in up-regulating the activity of the $\mathrm{N}$-methyl-D-aspartate receptors (NMDARs) at glutamatergic synapses (Yu et al. 1997; Salter and Kalia 2004). The NMDAR is a heterodimer of NR1 and NR2 subunits. The NR1 subunit is essential for pore formation and for the binding of several regulatory ligands, whereas NR2 subunits (NR2A-D) bind glutamate and determine the kinetic characteristics of specific NMDAR multimers (Monyer et al. 1994). Unlike the NR1 subunit, NR2A and NR2B are tyrosine phosphorylated by Src family kinases (Lau and Huganir 1995).

Recent studies have shown that NMDARs are subject to rapid activity-dependent trafficking between synaptic and extrasynaptic domains (Tovar and Westbrook 2002; Groc et al. 2006; Jeyifous et al. 2009) and that this trafficking is dependent on subunit tyrosine phosphorylation (Goebel et al. 2005; Snyder et al. 2005; Braithwaite et al. 2006). Localization of NMDAR to the postsynaptic membrane depends on NR2B phosphorylation at tyrosine 1472 (Nakazawa et al. 2002, 2006; Zhang et al. 2008). While both Src family kinases Src and Fyn phosphorylate NMDARs, NR2B surface expression is regulated by clathrin-mediated endocytosis through a cyclin-kinase 5 (Cdk5)dependent pathway that is regulated mainly by Src (Zhang et al. 2008).

The circuits mediating auditory fear conditioning consist of auditory and somatosensory inputs that converge onto neurons within the lateral nucleus of the amygdala (LA) (LeDoux 2000). The LA then projects to the central nucleus of the amygdala (CE), both directly and indirectly through the basolateral amygdala complex (BLA). Outputs from the central nucleus to the hypothalamus and brain stem then control the expression of fear responses such as freezing (Sigurdsson et al. 2007).

\footnotetext{
${ }^{3}$ Corresponding author.
}

E-mail sinai@lunenfeld.ca; fax (416) 586-4767.

Article is online at http://www.learnmem.org/cgi/doi/10.1101/Im.1765710.
Activation of NMDARs within the LA and BLA is necessary for the acquisition of fear memories (Gewirtz and Davis 1997; Lee and Kim 1998; Bauer et al. 2002). In addition, BLA and medial amygdala (MeA) activity are required for social recognition and affective responses to social cues (Fleming and Walsh 1994), and NMDAR antagonists disrupt social recognition (Gao et al. 2009). An increase in Fos expression, indicative of neuronal activity, has been observed in various amygdalar nuclei following both fear and social learning (Fleming and Walsh 1994; Kubota et al. 2004).

Here, we report that a Src peptide fragment ligated to the cell-permeable Tat motif Tat-Src (40-58), and previously shown to block the interaction of Src with the NMDARs (Gingrich et al. 2004), inhibited NR2B phosphorylation in vivo, decreased NR2B surface expression in cultured amygdalar neurons, decreased cued fear conditioning and social recognition, and impaired amygdalar long-term potentiation (LTP). These results demonstrate that Src can regulate amygdalar synaptic plasticity and learning by controlling the surface expression of NMDARs at amygdalar synapses

\section{Results}

Src inhibition decreases the phosphorylation of NR2B at Y1472 and increases the activity-dependent internalization of NR2B/NMDARs

To first assess if intravenously injected Tat-Src (40-58) peptide could accumulate in the amygdala, mice were injected with a Tat-Src (40-58) peptide conjugated with dansyl $1 \mathrm{~h}$ prior to amygdala dissection (Fig. 1). The dansyl epitope was detected in the amygdala of dansyl-Tat-Src (40-58)-injected mice but not in the amygdala of mice injected with saline (Fig. 1A). Furthermore, the tyrosine phosphorylation level of NR2B in Tat-Src (40-58)-injected mice demonstrated a significant decrease compared with saline-injected mice. Densometric quantification of the Western blots, probed using phospho antibody, 
A

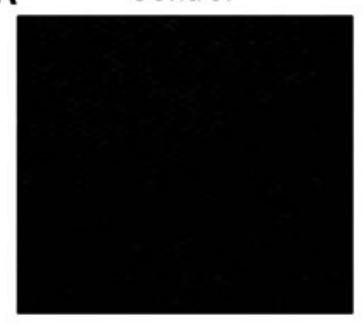

B

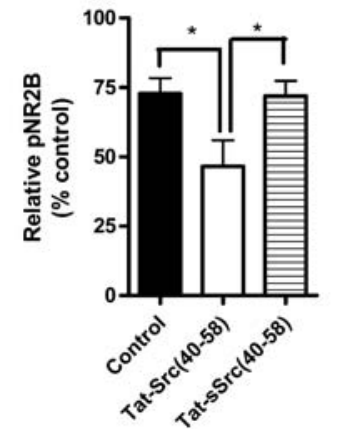

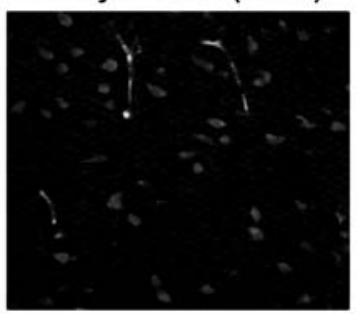

NR2B PY-1472 NR2B

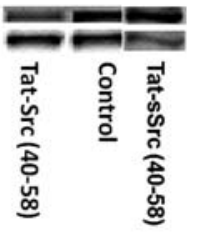

Dansyl-Tat-Src(40-58)

Figure 1. The Tat-Src (40-58) peptide penetrates the blood-brain barrier following i.v. injection and accumulates in amygdalar neurons where it inhibits NR2B phosphorylation. $(A)$ An antibody against dansyl detects stains in amygdala cells $1 \mathrm{~h}$ after the intravenous injection with dansyl-Tat-Src $(40-58)(9.3 \mathrm{nmol} / \mathrm{g})$ while no staining was observed in the amygdala of saline-injected mice. (B) Preinjection of Tat-Src $(40-58)$ decreases phosphorylation of NR2B at residue $\mathrm{Y} 1472$ in the amygdala. The level of phosphorylation of NR2B at residue Y1472 was monitored in amygdala tissue $1 \mathrm{~h}$ after i.v. injection with Tat-Src $(40-58)$ at $9.3 \mathrm{nmol} / \mathrm{g}$ or saline. Densiometric quantification demonstrated that Tat-Src (40-58) treatment decreased the phosphorylation of $Y 1472$ compared with control $\left({ }^{*} P<0.05\right)$.

showed that Tat-Src (40-58) treatment decreases the basal phosphorylation of Y1472 NR2B compared with control (control intensity: $72.76 \pm 5.59, n=7$; Tat-Src [40-58]: $46.51 \pm 9.462$, $n=7 ; \quad P<0.05$, Tat-sSrc [40-58]: 71.88 $\pm 5.447, \quad n=8 ; \quad P<$ $\left.0.05, F_{(1,19)}=0.026\right)$ (Fig. 1B). Thus, the inhibitory peptide accumulates in the amygdala sufficiently to reduce Src-dependent phosphorylation of NR2B.

Because inhibition of Src decreased phospho-Y1472 NR2B in the amygdala, we predicted that inhibition of Src interaction with the NMDAR complex should also result in an increased NMDARs endocytosis (Zhang et al. 2008). To study the effect of Src inhibition on NMDA receptor internalization, we examined the effects of this peptide on NMDA subunit surface expression in neuronal cultures from the amygdala (Fig. 2). Cultures were treated with peptide $(20 \mu \mathrm{M})$ or saline for $1 \mathrm{~h}$, fixed, and then immunostained with an NR1 antibody that recognizes an extracellular epitope. This surface NR1 immunostaining was followed by immunostaining with an antibody to NR2B (Fig. 2B). We then used both intensity of the surface colocalizatin (Pearson's correlation) and the number of dendritic puncta containing colocalized receptor subunits to quantify NR2B surface expression (Fig. 2C,D). There was a significant difference in surface colocalization between control and inhibitor-treated neurons (control: $0.76 \pm 0.00 \%, n=12$; Tat-Src [40-58], $0.53 \pm 0.01 \%, n=10 ; P<$ $0.001, F_{(1,21)}=166$, ANOVA) (Fig. 2C). Furthermore, in control cultures, $47.41 \pm 3.04(n=13)$ of NR2B puncta were also stained with NR1 antibody, whereas in cultures treated with the peptide inhibitor only $23.01 \pm 1.81(n=10)$ of NR2B puncta were also positive for NR1 $\left(P<0.001, F_{(1,22)}=40.59\right.$, ANOVA) (Fig. 2D).
Together these studies indicate that Src regulates the surface expression of NR2B-containing (i.e., functional) NMDAR multimers in the amygdala.

\section{Src inhibition impairs cued fear conditioning}

Cued fear conditioning is the most widely used behavioral task to assess amygdalar function (Phelps and LeDoux 2005). Inhibitor-injected mice showed impaired fear-related learning in cued fear conditioning. Mice were injected $1 \mathrm{~h}$ prior to a single pairing of a tone (conditioned stimulus, CS) and a foot shock (unconditioned stimulus, US) on the conditioning day. Prior to pairing, there were no significant differences between groups in baseline freezing (control: $1.85 \pm 0.84, n=13$; Tat-Src [40-58]: $0.97 \pm 0.49, n=12$; Tat-sSrc [40-58]: $3.33 \pm 1.51, n=8, P>0.1$, $F_{(2,30)}=1.48$, ANOVA), freezing in response to unpaired tone (control: $5.38 \pm 1.94, n=13$; Tat-Src [40-58]: $3.33 \pm 1.47, n=$ 12; Tat-sSrc [40-58]: $7.50 \pm 3.97, n=8, P>0.2, F_{(2,30)}=00.72$ ANOVA), or in post-shock freezing (control: $28.46 \pm 6.71, n=$ 13; Tat-Src [40-58]: $26.67 \pm 6.59, n=12$; Tat-sSrc [40-58]: $24.58 \pm 8.01, n=8 ; P>0.5, F_{(2,30)}=0.07$, ANOVA) (Fig. 3A), suggesting that the peptide did not alter general anxiety. At $24 \mathrm{~h}$ after pairing, the mice were tested for long-term contextual and cued fear memory. Subjects were placed in a novel chamber for $3 \mathrm{~min}$ prior to the presentation of the tone; both control and Tat-Src (40-58)-injected mice displayed only weak freezing in the novel chamber (control: $9.13 \pm 1.56, n=16$; Tat-Src [40-58]: $5.07 \pm 1.69, n=15$; Tat-sSrc [40-58]: $4.37 \pm 2.61, n=$ $8, P>0.1, F_{(2,36)}=2.02$, ANOVA) (Fig. 3B). During the tone delivery, Tat-Src (40-58)-injected mice demonstrated much weaker freezing $(33.33 \pm 4.388, n=15)$ in comparison with mice injected
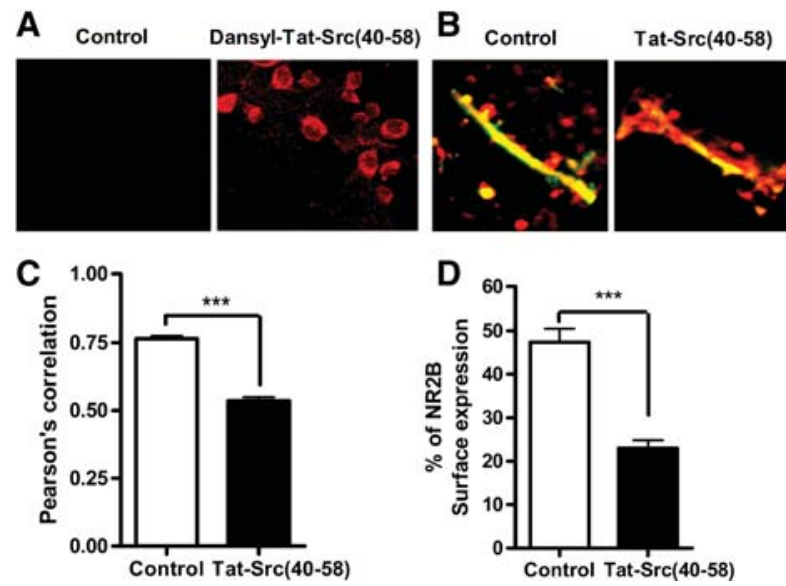

Figure 2. The Tat-Src (40-58) peptide accumulates intracellularly in cultured amygdala neurons and inhibits surface expression of NR2B. ( $A$ ) An antibody against dansyl detects dansyl-Tat-Src (40-58) $(20 \mu \mathrm{M})$ in amygdala neurons $1 \mathrm{~h}$ after treatment. No dansyl staining was observed in saline-treated cultures. (B) The surface expression of NR2B was evaluated in neuronal amygdala cultures treated for $1 \mathrm{~h}$ with control medium or with medium containing $20 \mu \mathrm{M}$ Tat-Src (40-58). Neurons were immunostained for NR1 (green) to visualize surface NMDARs before being permeabilized and immunostained for NR2B (red). An overlay of NR1 and NR2B (yellow) immunostaining reveals colocalization of NR1 with NR2B. Quantification of NR1/NR2B colocalization. For the statistical analysis, confocal images from 10-12 cells were collected at $100 \times$ magnification. All data are presented as mean \pm SEM of Pearson's correlation (percent) normalized to control $(* * * P<0.001$, ANOVA). Quantification of NR2B internalization. $(C, D)$ There was a significant decrease in NR2B surface expression in Tat-SrC (40-58)-treated neurons as determined by the number of puncta containing NR1 and NR2B $\left({ }^{* * *} P<0.001, \mathrm{ANOVA}\right)$. 
A

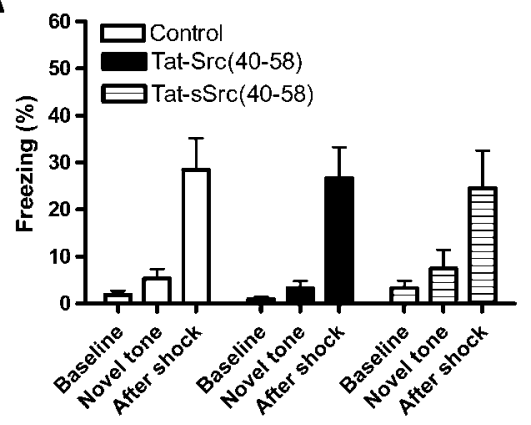

B

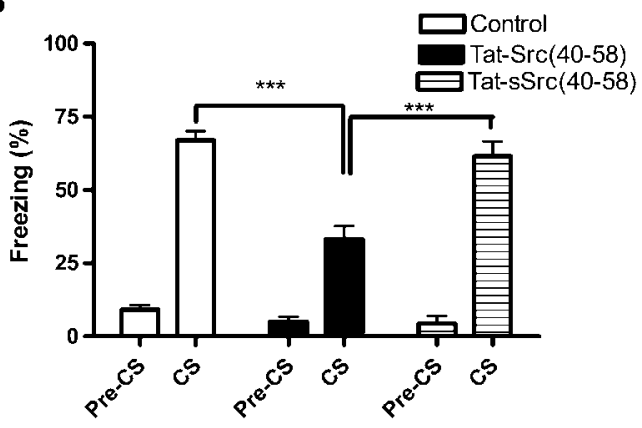

Figure 3. Preinjection of Tat-Src (40-58) impaired auditory fear conditioning at $24 \mathrm{~h}$ post-training but did not affect baseline responses to novel context, novel tone, or foot shock (general anxiety). (A) Freezing (as a percentage of observation time) in a novel conditioning chamber (Baseline) for saline-injected (open bars), Tat-Src (40-58)-injected mice (black bars), and Tat-sSrc (40-58)-injected mice (striped bars). After recording the baseline freezing for $120 \mathrm{sec}$, a tone was presented for 30 sec (novel tone), after which mice were given a foot shock and left inside the chamber for another $30 \mathrm{sec}$ (After shock). (B) Cued fear memory $24 \mathrm{~h}$ after pairing. Pre-CS is freezing in the absence of the tone (Pre-CS) in a novel context while CS is percentage freezing during tone presentation $\left({ }^{* * *} P<0.005\right.$, ANOVA).

with saline $(66.98 \pm 3.126, n=16, P<0.0001)$ and Tat-sSrc $(40-$ 58) (Tat-sSrc [40-58]: 61.46 $\pm 5.20, n=8 P<0.0007, F_{(2,36)}=$ 21.63, ANOVA) (Fig. 3B), suggesting that Src inhibition significantly decreased long-term memory of the CS-US association. In contrast, contextual fear was not affected by Src inhibition (control: $37.80 \pm 6.78, n=13$; Tat-Src [40-58]: $32.59 \pm 6.03, n=$ 12 and Tat-sSrc [40-58]: $28.42 \pm 5.05, n=8 ; P>0.5, F_{(2,30)}=$ 0.5, ANOVA) (Fig. 4A). It is also possible that Src inhibition decreases expression, rather than formation, of auditory fear memory. To examine this, we trained mice for auditory fear conditioning as before but injected Tat-Src (40-58) and Tat-sSrc (4058) $60 \mathrm{~min}$ before auditory testing rather than before training. Figure $4 \mathrm{~B}$ shows that injecting the inhibitor before testing does not decrease the expression of an auditory fear memory.

Freezing levels in both groups increased during the tone presentation (Tat-Src [40-58]: $62.12 \pm 6.330 n=9$ and Tat-sSrc [40-58]: $55.89 \pm 4.885 n=9 ; P>0.5, F_{(1,16)}=0.61$, ANOVA). Therefore, Src inhibition does not decrease expression of an auditory fear memory (Fig. 4B).

Because pain sensitivity can also affect the strength of associative learning, we measured the pain threshold in two different tasks. First, nociceptive response was assessed using the tail-flick test, which engages supra-spinal pathways of the nociceptive system. Second, the minimum foot-shock intensity required to produce an audible vocalization response was determined. As shown in Table 1, tail-flick latencies did not differ significantly between the groups; neither one-way ANOVA nor Tukey's post-hoc analysis revealed a difference between the two groups (control: $3.87 \pm$ $0.51, n=8$; Tat-Src [40-58]: $3.62 \pm 0.56, n=8, P>0.5, F_{(1,15)}=$ 0.11 , ANOVA). Similarly, the shock threshold for vocalization was unaltered (control: $0.26 \pm 0.01 \mathrm{~mA}, n=8$; Tat-Src [40-58]: $0.26 \pm 0.01, n=8, P>0.5, F_{(1,15)}=0.26$, ANOVA).

\section{Src inhibition impairs social recognition}

To further investigate the role of Src in amygdalar learning, we studied a second amygdalar-dependent task that does not rely on aversive stimulation, social recognition (Richter et al. 2005). Mice were injected $45 \mathrm{~min}$ prior to a training trial that consisted of a 15-min habituation to a new home cage, followed by a 2-min presentation of a novel juvenile (Presentation 1, P1). The social interaction time was measured, after which the animals were returned to their home cages. Mice were then tested $1 \mathrm{~d}$ after this initial interaction using the same juvenile (Presentation 2, P2). There was a significant reduction in the duration of investigation time in the saline-injected control group but not in the peptide-injected group, indicating that control mice were better able to recognize the pre-exposed juvenile (control P1: $71.61 \pm$ $6.25 \mathrm{sec}, n=10$; control P2: $46.90 \pm 6.23 \mathrm{sec}, n=10 ; P=0.007$, ANOVA, $\left.F_{(1,19)}=8.96\right)$ (Tat-Src [40-58] P1: $58.47 \pm 4.33 \mathrm{sec}, n=$ 9; inhibitor P2: $\left.53.31 \pm 7.24 \mathrm{sec}, n=9, P>0.5, F_{(1,17)}=3.81\right)$ (Tat-sSrc [40-58] P1: $75.14 \pm 3.91 \mathrm{sec}, n=8$; Tat-sSrc [40-58] P2: $\left.43.89 \pm 5.23 \mathrm{sec}, n=8, P=0.003, F_{(1,15)}=22.87\right)$ (Fig. $5 \mathrm{~A}$ ). As a control, we examined the response to a novel (non-preexposed) juvenile presented after P2. In this test, there was no significant reduction in the investigation duration

\section{A}

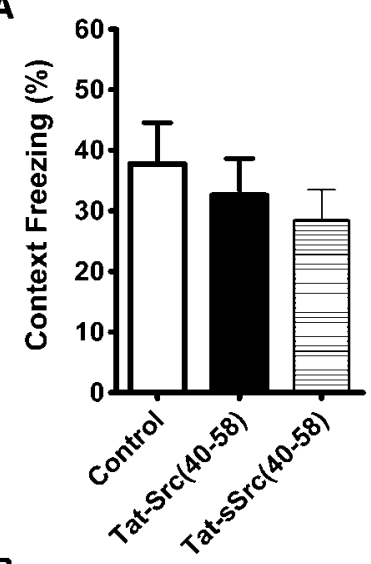

B

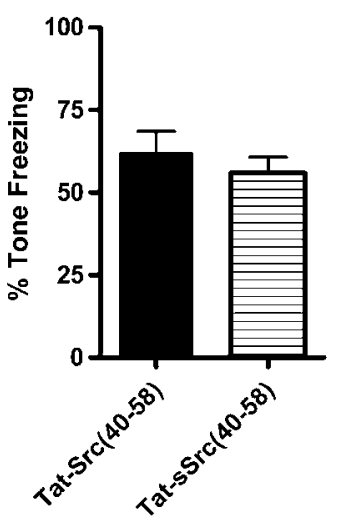

Figure 4. The effects of Src inhibition are specific to the formation of auditory fear memory. Src inhibition does not impair the formation of a context fear memory $(A)$ or expression of an auditory fear memory $(B)$. 
Table 1. Nociception and vocalization threshold in control and Tat-Src (40-58)-injected mice

\begin{tabular}{lcc}
\hline & Control & Tat-Src (40-58) \\
\hline Tail-flick latency (sec) & $3.87 \pm 0.51 n=8$ & $3.62 \pm 0.56 n=8$ \\
Vocalization threshold (mA) & $0.26 \pm 0.00 n=8$ & $0.26 \pm 0.00 n=8$
\end{tabular}

Tail-flick latencies and shock threshold to vocalization were not affected by Src inhibition. Data presented are average ( $\pm \mathrm{SE}), n=8$ for each group.

(control: $74.41 \pm 6.74 \mathrm{sec}, n=9, P>0.6$, ANOVA $F_{(1,19)}=0.5$; Tat-Src [40-58]: $64.65 \pm 4.44 \mathrm{sec}, n=10, P>0.6, F_{(1,17)}=8.7$ ANOVA and Tat-sSrc [40-58]: $74.00 \pm 6.857 \mathrm{sec}, n=8, P>0.8$ $F_{(1,15)}=0.02$ ) (Fig. 5B), indicating that the reduction at P2 was specific to familiar juveniles. Thus, inhibitor injected mice are unable to form robust long-term memories for social recognition.

Impaired synaptic plasticity in inhibitor incubated slices A long-lasting Hebbian synaptic potentiation (LTP) within lateral (LA) and basolateral (BLA) nuclei is a key neurocellular sequela of cued fear conditioning (Maren 1999b). Therefore, we examined LTP in the amygdala in vitro following 1-h preincubation with either saline or $20 \mu \mathrm{M}$ Tat-Src [40-58]. In saline-treated slices, tetanic stimulation (consisting of five, 1-sec trains of $100 \mathrm{~Hz}$ ) to the

A

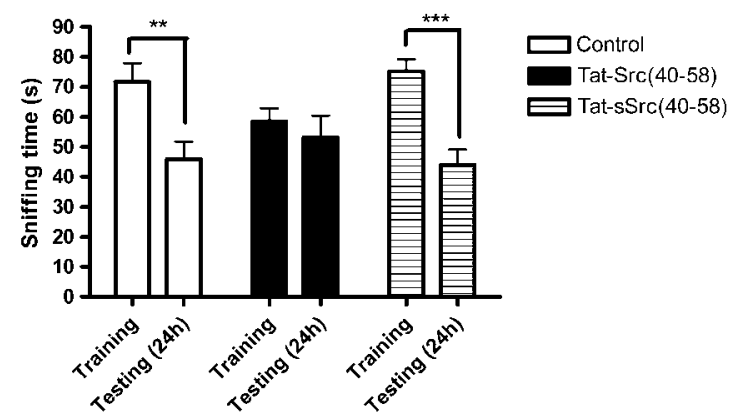

B

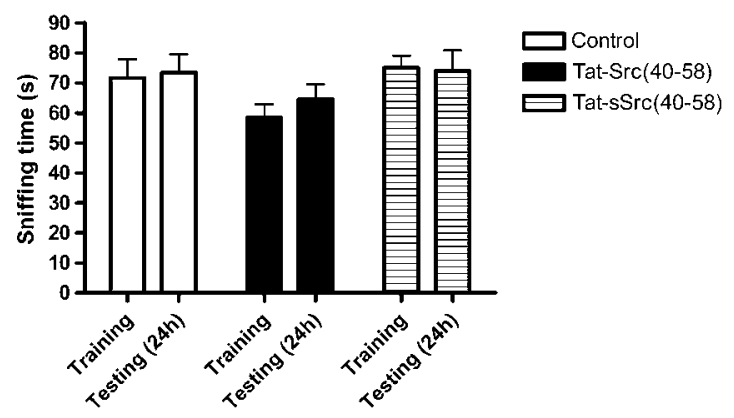

Figure 5. Long-term social memory is impaired in inhibitor-injected mice. Social recognition was assessed in two successive 2-min interaction trials with intertrial intervals of $24 \mathrm{~h}$. On the training day, mice were injected $45 \mathrm{~min}$ prior to training. Mean investigation duration is shown for training and test trials. (A) Familiar juveniles were exposed to both groups at test trials. There was significant reduction in investigation duration, indicating long-term social memory in the saline-injected and Tat-sSrc (40-58)-injected mice (open bars, $* * P<0.005$ and striped bars, $\left.{ }^{* * *} P<0.005\right)$ but not in the Tat-Src $(40-58)$-injected group (solid bars). (B) Novel juveniles were exposed to both groups after the test trial; there was no significant difference in interaction times between these second novel juveniles and those present $24 \mathrm{~h}$ earlier.
LA-BLA pathway induced a long-lasting potentiation (at $55-60$ min post-tet: $166 \pm 14 \%$ of baseline) (Fig. 6). In contrast, LTP was greatly diminished in slices preincubated with peptide (at $55-60 \mathrm{~min}$ post-tet: $119 \pm 5 \%$ of baseline; $P<0.02$ ) while the early post-tetanic potentiation (PTP) was unaltered. Furthermore, Tat-Src (40-58) caused no significant reduction in the baseline population EPSP slope prior to tetanization (control slices: $0.75 \pm 0.07 \mathrm{mV} / \mathrm{ms}$; peptide-treated slices: $0.66 \pm$ $0.10 \mathrm{mV} / \mathrm{ms}$ ). For these data we conclude that Src activity is required for the LTP at LA to BLA synapses but not for basal neurotransmission.

\section{Discussion}

Blocking the interaction of the Src tyrosine kinase with the NMDAR complex impaired auditory conditioned fear memory and social recognition. Inhibition of Src-NMDAR interaction also attenuated NR2B phosphorylation and decreased NR2B surface expression in the amygdala. Furthermore, at the lateral to basolateral nucleus pathway (LA-BLA), inhibition of Src impaired LTP, a form of synaptic plasticity that is vital for encoding CS-US associations (Maren et al. 2001). In sum, these data indicate that Src-mediated phosphorylation of NMDARs is necessary for two vital forms of amygdala-dependent learning.

The amygdale is essential for both the encoding and storage of fear memories (Campeau and Davis 1995; LeDoux 2000). Sensory information encoding the US and CS is conveyed by glutamatergic projections that terminate on neurons within both the basolateral and lateral nuclei (Fanselow and LeDoux 1999; Maren 1999a). Neurons within the LA and BLA exhibit short-latency firing during US and CS presentation (Maren et al. 1991; Quirk et al. 1995). Temporal pairing of the US and CS can induce LTP of CS-evoked AMPA-mediated synaptic responses both in vitro and in vivo (McKernan and Shinnick-Gallagher 1997), suggesting that NMDAR-dependent Hebbian synaptic plasticity mediates the US-CS association at the neuronal level. Similarly, tetanic stimulation also induces a form of LTP that can be blocked by NMDAR antagonists (Gean et al. 1993; Maren and Fanselow 1995). The NR2B subunit is expressed on the majority of thalamoamygdala dendritic spines (Radley et al. 2007), and antagonism specific to NR2B-containing NMDARs can block both cued fear memories and synaptic potentiation ( $\mathrm{Li}$ et al. 1995; Bauer et al. 2002; Nakazawa et al. 2006). Significantly, mice lacking the Src phosphorylation site Y1472 of NR2B also show deficit fear learning (Nakazawa et al. 2002, 2006). Thus, our results underscore the importance of the Src-NR2B interaction in the regulation of amygdalar synaptic plasticity and learning. By controlling the surface expression of NR2B-containing NMDARs, Src-mediated phosphorylation regulates the threshold for NMDA-dependent synaptic plasticity and learning. Indeed, blockade of conditioned fear and LTP suggest that basal Src activity is necessary for proper expression of these behaviors, possibly by maintaining sufficient numbers of postsynaptic NMDA receptors.

The peptide Src inhibitor Tat-Src (40-58) was injected systemically so as to avoid confounds of neural damage associated with direct needle injection into the amygdala. Thus, while Tat-Src (40-58) blocked LTP in the LA-BLA pathway, it is possible that disrupted transmission or plasticity within other circuits, either within or outside the amygdala, could contribute to the observed deficits in conditioned fear. A decrease in nociceptive transmission could interfere with encoding of the US-CS association; alternatively, suppression of amygdale output or effects within those brain stem nuclei controlling fear behavior may also account for the observed response. The attenuation of the 


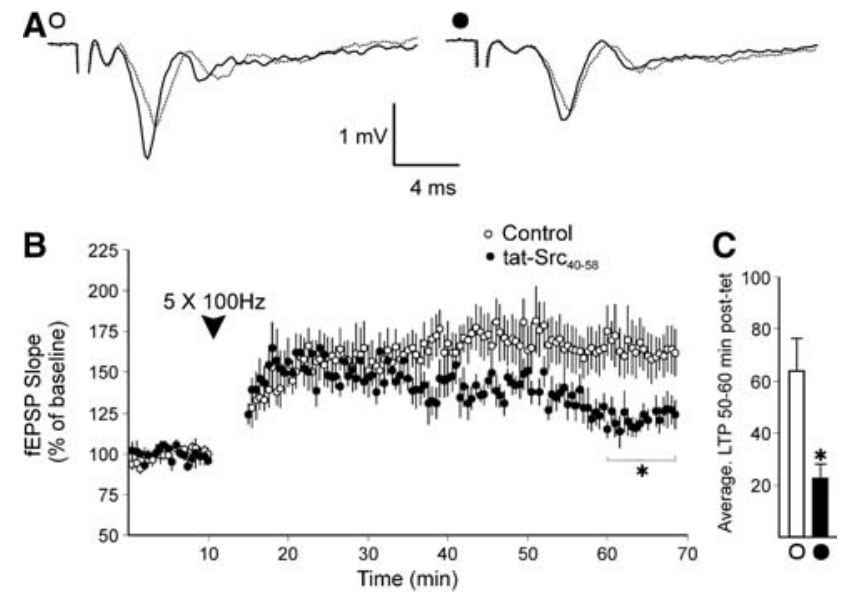

Figure 6. Incubation in the Src inhibitory peptide Tat-Src (40-58) markedly reduced LTP in the lateral to BLA pathway in vitro. $(A)$ Sample field excitatory postsynaptic potentials (fEPSPs) recorded 1 min before and 60 min following tetanization for a control slice (demarcated by a closed circle) and a Tat-Src (40-58)-treated slice (open circle). (B) Average fEPSP slope for control (closed circles) and Tat-Src (4058)-treated slices (open circles) as a percentage of baseline fEPSP slope. While tetanic stimulation induced similar initial increase in fEPSP slope, LTP decayed to near baseline in the presence of Tat-Src (40-58). (C) Average LTP from each slice determined from the 20 sample sweeps recorded $50-60$ min post-tetanus $\left({ }^{*} P<0.02\right)$.

freezing response in inhibitor- injected mice was not due to a deficit in pain perception, however, because injected mice have had normal post-shock freezing, freezing threshold, and tail withdrawal latency to hot water. Second, inhibitor-injected mice exhibited no impairment in context-dependent freezing relative to controls, indicating that downstream synaptic pathways mediating the freezing response were not markedly affected. Third, Src inhibition did not alter expression of an auditory fear memory. Therefore, the auditory fear memory impairment cannot be attributed to pain perception, an increase in overall fear memory, a nonspecific increase in anxiety, or a general change in auditory processing.

Social recognition tasks take advantage of the innate tendency of an adult rodent to investigate nonfamiliar over familiar conspecifics and, like fear conditioning, is amygdalar dependent (Meredith and Westberry 2004; Markham and Huhman 2008; Petrulis 2009). C-Fos expression, which is indicative of neuronal activity, is enhanced in the basolateral and medial amygdala following social recognition tasks (Borelli et al. 2009). In the present study, mice were not able to form a robust long-term memory for social recognition following inhibition of the Src-NMDAR interaction. Thus, it is possible that NMDA-dependent plasticity within the BLA could facilitate social recognition. It is well known that an increase in synaptic strength lasting longer than 1-3 $\mathrm{h}$, termed L-LTP, requires protein synthesis in amygdala (Huang and Kandel 2007) and that protein synthesis inhibitors block long-term (but not short-term) social recognition (Richter et al. 2005). Given that the Src inhibitor was injected systemically, however, it is again possible that Src inhibition within other pathways in the amygdala contributes to the observed deficits in social memory, particularly as we have not investigated synaptic plasticity of NR2B trafficking in the medial nucleus. Local injections of the inhibitor into the medial amygdala and other relevant pathways are required to isolate the relevant loci.

The Src kinase is anchored within the NMDAR complex through an adaptor protein called NADH dehydrogenase subunit (ND2) (Gingrich et al. 2004). Blocking the interaction between the
Src unique domain and ND2 releases Src from the NMDAR complex, thereby inhibiting Src-mediated phosphorylation. To understand the neurocellular mechanisms and role of this inhibition in amygdala learning, we measured NR2B phosphorylation levels in the amygdala of inhibitor-injected mice and NR2B internalization in primary amygdala cultures. There was a decrease in NR2B Tyr-1472 phosphorylation $1 \mathrm{~h}$ after injection, while NR2B surface expression was decreased in amygdala cultures in the same time frame. Moreover, the number of membrane clusters coexpressing NR1 and NR2B was reduced, indicating that Tyr-1472 phosphorylation is necessary for proper localization of NMDARs at postsynaptic sites. Whether Tyr-1472 phosphorylation by Src directly regulates NR2B endocytosis is not known, but one possibility is that phosphorylation of Y1472 NR2B down-regulates the binding of NR2B to the endocytosis motif AP-2. The $\mathrm{C}$ terminus of the NR2B subunit contains a clathrin adaptor AP-2 binding site and YEKEL internalization motif. Tyrosine-1472 is within this motif; moreover, tyrosine phosphorylation inhibits the binding of AP-2 and promotes surface expression of NMDARs (Prybylowski et al. 2005; Zhang et al. 2008). Although Fyn, not Src, has been suggested to be the major tyrosine kinase responsible for the phosphorylation of NR2B (at least in transferred HEK cells) (Nakazawa et al. 2001), NR2B phosphorylation is not abolished in Fyn knockout mice. This suggests that Src family tyrosine kinases other than Fyn can also phosphorylate NR2B. Indeed, NR2B phosphorylation is modulated by cycline-dependent kinase IV (Cdk4) and PSD-95 in a Src-dependent but Fyn-independent manner (Zhang et al. 2008), suggesting that Src may be responsible for the remaining phosphorylation of NR2B in Fyn mutant mice.

These results provide a plausible mechanism for the regulation of amygdala-dependent learning by the Src protein kinase. By maintaining NR2B surface localization on the postsynaptic dendrites of amygdalar neurons within the LA and BLA, Src supports the induction of LTP concomitant with US-CS pairing. Further illumination of the role of Src signaling in amygdalar learning could facilitate the development of novel treatments for psychiatric diseases characterized by aberrant fear responses.

\section{Materials and Methods}

\section{Subjects}

Adult C57BL/6J mice were purchased from Jackson Laboratory (Bar Harbor, ME) (8-14 wk old). Male juvenile mice of the same background were obtained from our own breeding colony (25$30 \mathrm{~d}$ old). Mice were housed in groups of five in transparent cages on wood chip bedding and provided with dry food pellets and water ad libitum. They were maintained on a 12:12-h light-dark artificial cycle (lights on from 05:00 to 17:00) in a temperature$\left(20^{\circ} \mathrm{C} \pm 1{ }^{\circ} \mathrm{C}\right)$ and humidity $(50 \%-60 \%)$-controlled vivarium. One week before the beginning of the experiments, the mice were handled for $2 \mathrm{~min}$ each day and habituated to the restraining tube used for i.v. injections. Mice were tested during the light phase between 08:00 and 16:00 h. All animal procedures were approved by the Animal Management Committee of Mount Sinai Hospital and strictly followed the requirements of the Province of Ontario Animals for Research Act 1971 and the Canadian Council on Animal Care.

\section{Drugs}

Tat-Src (40-58), Tat-sSrc (40-58), and dansyl-Tat-Src (40-58) were synthesized at the hospital for Sick Children Advanced Protein Technology Centre (Toronto, Canada) on a NovasynCrystal Peptide Synthesizer (NovaBiochem) by Fmoc-chemistry on PEG-PS resin. The solvent used throughout the synthesis was freshly purified DMF. A solution of $20 \%$ piperidine in DMF was used for the removal of the Fmoc-protection group. For each 
$0.5 \mathrm{~g}$ of resin $(0.1 \mathrm{mmol}$ substitution) $4 \times$ excess of Fmoc amino acid activated with HATU and diisopropylethylamine $(1: 1: 2 \mathrm{~mol} / \mathrm{mol} / \mathrm{mol})$ was used for the coupling reaction. The reaction time was $1 \mathrm{~h}$ at room temperature. After the synthesis, the peptide-resine conjugate was washed $2 \times$ with iso-propanol, and another $2 \times$ with diethyl ether, and dried under reduced pressure. The dry peptide-resins conjugate was treated with $20 \mathrm{~mL}$ of TFA containing $4 \mathrm{~mL}$ thioanisole, $0.4 \mathrm{~mL} \mathrm{~m}$-cresol, $2 \mathrm{~mL} \mathrm{1,2-ethanedithiol,} \mathrm{and} 4 \mathrm{~mL}$ bromotrimethylsilane at $0^{\circ} \mathrm{C}$ for $2 \mathrm{~h}$. After removal of the resine by filtration and TFA under reduced pressure, $50 \mathrm{~mL}$ of diethyl ether and $50 \mathrm{~mL}$ of $0.1 \%$ TFA solution were added to the residue. The aqueous layer was extracted $3 \times$ with diethyl ether and freeze-dried. The crude peptide was dissolved in 0.1 TFA and desalted on a Sephadex G10 column. The peptide was then analyzed by reverse-phase HPLC on a Bondclone C18 column (Phenomenex). Mass spectrometry analysis was performed on a Biosystems/MDS Sciex MALDI Qstar XL quadropole time-of-flight (QqTOF) instrument. The peptides were dissolved in saline and administered i.v. at a dose of $(9.3 \mathrm{nmol} / \mathrm{g}) 60 \mathrm{~min}$ before the training phase or testing phase of each experiment. Dose and route of administration was chosen in a pilot experiment where dansyl-tagged Tat-Src (40-58) reaches peak concentration $75 \mathrm{~min}$ after an i.v. injection. Concentrations of the inhibitor would reach undetectable levels in the brain by $24 \mathrm{~h}$ post-injection.

\section{Immunohistochemistry}

Polyclonal antibodies against phospho Tyr-1472 and NR2B were a kind gift from Takanobu Nakasawa (Nakazawa et al. 2006). Preparation of lysates from amygdala slices, immunoprecipitation, and immunoblotting were performed as described previously (Nakazawa et al. 2001, 2002). For quantification, the immunoreacted protein bands were analyzed with the Biorad image software.

\section{Dissociated neuronal amygdala cultures}

Pregnant mice were sacrificed by cervical dislocation and dissociated neuronal amygdala cultures were prepared from postnatal day 1 (P1) mouse brain. Dissociated amygdala neurons were plated on poly-D-lysine-coated glass coverslips at a density of $115,000 / 25-\mathrm{mm}$ coverslip. Neurons were grown in Neurobasal medium supplemented with B27 and GlutaMax (Invitrogen) for 18-20 d in vitro (DIV). To analyze the effect of inhibition of Src, cultures were treated for $60 \mathrm{~min}$ with either $20 \mu \mathrm{M}$ Tat-Src (40-58) dissolved in saline or with saline (control).

\section{Immunocytochemistry}

In order to visualize NMDAR surface expression, neurons were fixed after treatment with 4\% paraformaldehyde, 5\% sucrose in PBS for 10 min, washed with PBS, and then blocked with $10 \%$ normal goat serum (NGS) in PBS for $1 \mathrm{~h}$. Surface NMDARs were stained overnight in 3\% NGS in PBS with a mouse antibody that recognizes an extracellular epitope of the NR1 subunit (NR1, 1:200; BD PharMingen). After washes in PBS, neurons were permeabilized with $0.2 \%$ Triton X-100 in PBS with $10 \%$ bovine serum albumin (BSA) and incubated overnight with a rabbit NR2B antibody (1:100; Millipore) to label both surface and internalized NR2B-containing NMDARs. After extensive washes in PBS with $0.1 \%$ Triton $X-100$, cultures were incubated with anti-mouse (green; Cy2) and anti-rabbit (red; Cy5) secondary antibodies (1:200; Jackson Immuno Research). Images were captured using a Leica DM LFSA confocal microscope under identical conditions for all preparations (100 objective, $1024 \times 1024$ pixels format, identical camera gain and black level). Two different methods were used to analyze the NR2B surface expression. First, the intensity (Pearson's correlation) was used to measure the colocalization intensity between surface NR1 and total NR2B. The method is described by Lavezzari et al. (2004). Second, the level of surface expression of NR2B-containing NMDARs was determined by quantifying the number of surface dendritic puncta that were immunostained for NR2B compared with the number of puncta immunostained for both NR2B and NR1, using the method described by Zhang et al. (2008). NR2B surface expression was defined as the ratio of surface receptor (NR2B + NR1) versus total receptor (NR2B). The degree of surface expression was expressed as percentage of mean \pm SEM normalized to control and statistically compared using a one-way ANOVA with post hoc between treatment groups analyzed with Tukey's post-hoc multiple comparison test (MINITAB 13 for Windows).

\section{Fear conditioning}

The fear conditioning apparatus (MED Associates Inc.) consisted of a test chamber ( $25 \mathrm{~cm}$ high, $30 \mathrm{~cm}$ wide, $25 \mathrm{~cm}$ deep), a removable grid floor of 36 stainless steel rods (3.2 mm diameter, $4.7 \mathrm{~mm}$ apart) connected to a constant current shock generator, and an amplifier and speaker. A 12-in, 8-W fluorescent tube (GE Lighting Canada) illuminated the chamber interior. A computer running automated fear conditioning software (FreezeFrame; Actimetrics Software) administered foot shocks and auditory tones. Video images were recorded from the chamber, and the activity of subjects was recorded throughout the experiment. Immediately prior to training, the chamber was cleaned with $70 \%$ ethanol. A white cloth covered the front of the chamber. Each subject was removed from its home cage and placed into the chamber for $2 \mathrm{~min}$ prior to conditioning. Activity during this time was recorded as baseline. Conditioning consisted of a single pairing of an auditory tone with a continuous foot shock. The tone $(3600 \mathrm{~Hz} ; 80 \mathrm{~dB})$ was delivered 2 min after the training session started and was $30 \mathrm{sec}$ in duration. The foot shock ( $0.75 \mathrm{~mA}$ scrambled) was presented during the last $2 \mathrm{sec}$ of the tone. The mouse was left in the chamber for $30 \mathrm{sec}$ and then returned to its home cage. Approximately $24 \mathrm{~h}$ later, each subject was returned to the chamber and percentage of freezing was monitored for $5 \mathrm{~min}$ in the conditioned context. The activity of each subject was recorded manually by an observer using a stop watch. The mean activity during the context exposure was calculated, subtracted by the mean baseline activity, and used as a measure of contextual learning. Two hours later, the context was changed by covering the grid floor with a sheet of white PerspexTM (polymethyl methacrylate), inserting two sheets of transparent Perspex into the chamber to give it a prism shape, cleaning the chamber with $1 \%$ vinegar, covering the front door with a striped black and white card, and turning on the ceiling lights. Each mouse was placed into the altered chamber and allowed $3 \mathrm{~min}$ for exploration in the novel environment, after which the auditory tone of 3-min duration was delivered. The mean activity during cue delivery was calculated, subtracted by the mean activity in the novel context (prior to the presentation of the tone), and was taken as a measure of cue-associated learning. The drugs were administered $60 \mathrm{~min}$ prior to training in each experiment.

Two different methods were used to measure pain threshold. Tail flick was measured by using a hot-water bath system. The water was maintained at $55^{\circ} \mathrm{C}$ in a constant-temperature water bath. Mice were held in a restrainer, and the distal third of the tail was immersed in the bath. The time required for a mouse to remove its tail was measured by use of a stopwatch, and the tailflick latency score was calculated as the mean of the last two of three trials. Trials were separated by 30 -sec intervals.

Shock threshold was assessed by placing the animal in the fear conditioning chamber and by delivering foot shocks starting at $0.075 \mathrm{~mA}$ and increasing by $0.05 \mathrm{~mA}$ every $30 \mathrm{sec}$. The experiment was terminated at the shock intensity sufficient to induce audible vocalization.

\section{Social recognition}

For social recognition experiments, we used the procedure described by Kogan et al. (2000). In brief, adult male mice were single-caged prior to an experimental session. After $15 \mathrm{~min}$ a male juvenile mouse was added to the cage for an initial interaction trial of $2 \mathrm{~min}$. Twenty hours later, either the same or a novel juvenile was placed back into the adult's cage for a 2-min test trial. 
Social investigation of the juvenile (mainly sniffing and licking of the anogenital region of the juvenile) by the adult mouse was observed continuously by an observer who scored the duration of investigation behavior with the Noldus software (Observer 5.0; Noldus Information Technology).

\section{Statistics for behavior tests}

Data from cued fear conditioning and social recognition were subjected to one-way ANOVA between treatment groups. When the ANOVA detected significant treatment effects, pairwise differences between means for a given variable were evaluated using Tukey's post-hoc multiple comparison test, with significance set at $P<0.05$. All statistics were calculated using MINITAB for Windows 13.32 (Minitab Inc.). All values reported in the text and figures are expressed as means \pm SEM.

\section{Electrophysiology}

Extracellular excitatory field potentials (fEPSPs) in the lateral nucleus to basolateral nucleus pathway (LA-BLA) were recorded as described by Schimanski and Nguyen (2005). In brief, freshly isolated brains from 8-10-wk-old C57Bl6/J mice were submerged in ice-cold artificial cerebrospinal fluid (ACSF) containing (in $\mathrm{mM}$ ): $126 \mathrm{NaCl}, 3.3 \mathrm{KCl}, 26 \mathrm{NaHCO}_{3}, 2 \mathrm{CaCl}_{2}, 2 \mathrm{MgCl}_{2}$, and 10 glucose $(\mathrm{pH} 7.35)$. Coronal slices $(400 \mu \mathrm{M})$ were prepared from brain sections using a vibratome. Slices were maintained at room temperature in a submerged chamber containing aerated ACSF $\left(95 \% \mathrm{O}_{2} / 5 \% \mathrm{CO}_{2}\right)$ for a minimum of $3 \mathrm{~h}$ before electrophysiological studies commenced. Individual slices were then transferred to a submerged recording chamber and superfused with aerated ACSF with the ionic composition as above except that $\mathrm{KCl}$ was raised to $4 \mathrm{mM}$ and $\mathrm{MgCl}_{2}$ reduced to $1.2 \mathrm{mM}$ (superfusion ACSF). Slices were maintained at $29.5^{\circ} \mathrm{C} \pm 0.5^{\circ} \mathrm{C}$. To measure fEPSPs in the LA-BLA pathway, a concentric bipolar stimulating electrode (platinum/iridium, HFC) was inserted into the LA near the apex formed by the intersection of the internal and external capsules. The recording electrode was placed ventrally at the LA-BLA boundary to measure field potentials from the dendrites of BLA neurons (Schimanski and Nguyen 2005). Electrophysiological responses induced by an S88 stimulator (Grass Instruments) were recorded using an Axopatch 1D amplifier (Axon Instruments). Only preparations showing a clearly discernable, early presynaptic volley followed by a fEPSP of at least $2 \mathrm{mV}$ maximum amplitude were used. The fEPSP slope, measured between $\approx 10 \%-60 \%$ of the peak amplitude, was used to estimate synaptic strength. Initially, slices were superfused in an open bath, but switched to a closed recirculating bath containing $40 \mathrm{~mL}$ superfusion ACSF with $10 \mu \mathrm{M}$ bicuculine, and in experimental trials, with $20 \mu \mathrm{M}$ of the Src inhibitory peptide Tat-Src (40-58). These drug-treated slices were preincubated with peptide for 40-50 min before the start of baseline recording, while control slices were preincubated in ACSF and bicuculine in the closed bath for the same duration. In both groups, the chamber remained in the closed configuration for the remainder of the experiment. Following preincubation, fEPSPs at $50 \%$ of maximum slope were recorded every $30 \mathrm{sec}$ for $15-20 \mathrm{~min}$. The last $10 \mathrm{~min}$ of this recording period was used as the baseline for subsequent LTP measurements. To induce LTP, the LA was then tetanized by five $100-\mathrm{Hz}$ trains lasting $1 \mathrm{sec}$ and separated by $10 \mathrm{sec}$. Post-tetanic fEPSPs were recorded once every $30 \mathrm{sec}$ for the next $60 \mathrm{~min}$.

\section{Acknowledgments}

We thank John Georgiou for technical support, Mike Salter for advice, and Tatiana Lipina and Greer Kirshenbaum for reading the manuscript. This study was supported by grants-in-aid to J.C.R. from the Canadian Institutes of Health Research (CIHR; grant no. MOP-13239) and the OSOTF studentship award from Samuel Lunenfeld Research Institute.

\section{References}

Bauer EP, Schafe GE, LeDoux JE. 2002. NMDA receptors and L-type voltage-gated calcium channels contribute to long-term potentiation and different components of fear memory formation in the lateral amygdala. J Neurosci 22: 5239-5249.

Borelli KG, Blanchard DC, Javier LK, Defensor EB, Brandao ML, Blanchard RJ. 2009. Neural correlates of scent marking behavior in C57BL/6J mice: Detection and recognition of a social stimulus. Neuroscience 162: 914-923.

Braithwaite SP, Adkisson M, Leung J, Nava A, Masterson B, Urfer R, Oksenberg D, Nikolich K. 2006. Regulation of NMDA receptor trafficking and function by striatal-enriched tyrosine phosphatase (STEP). Eur J Neurosci 23: 2847-2856.

Campeau S, Davis M. 1995. Involvement of the central nucleus and basolateral complex of the amygdala in fear conditioning measured with fear-potentiated startle in rats trained concurrently with auditory and visual conditioned stimuli. J Neurosci 15: 2301-2311.

Fanselow MS, LeDoux JE. 1999. Why we think plasticity underlying Pavlovian fear conditioning occurs in the basolateral amygdala. Neuron 23: 229-232.

Fleming AS, Walsh C. 1994. Neuropsychology of maternal behavior in the rat: C-fos expression during mother-litter interactions. Psychoneuroendocrinology 19: 429-443.

Gao XM, Elmer GI, Adams-Huet B, Tamminga CA. 2009. Social memory in mice: Disruption with an NMDA antagonist and attenuation with antipsychotic drugs. Pharmacol Biochem Behav 92: 236-242.

Gean PW, Chang FC, Huang CC, Lin JH, Way LJ. 1993. Long-term enhancement of EPSP and NMDA receptor-mediated synaptic transmission in the amygdala. Brain Res Bull 31: 7-11.

Gewirtz JC, Davis M. 1997. Second-order fear conditioning prevented by blocking NMDA receptors in amygdala. Nature 388: 471-474.

Gingrich JR, Pelkey KA, Fam SR, Huang Y, Petralia RS, Wenthold RJ, Salter MW. 2004. Unique domain anchoring of Src to synaptic NMDA receptors via the mitochondrial protein NADH dehydrogenase subunit 2. Proc Natl Acad Sci 101: 6237-6242.

Goebel SM, Alvestad RM, Coultrap SJ, Browning MD. 2005. Tyrosine phosphorylation of the $N$-methyl-D-aspartate receptor is enhanced in synaptic membrane fractions of the adult rat hippocampus. Brain Res Mol Brain Res 142: 65-79.

Groc L, Heine M, Cousins SL, Stephenson FA, Lounis B, Cognet L, Choquet D. 2006. NMDA receptor surface mobility depends on NR2A-2B subunits. Proc Natl Acad Sci 103: 18769-18774.

Huang YY, Kandel ER. 2007. Low-frequency stimulation induces a pathway-specific late phase of LTP in the amygdala that is mediated by PKA and dependent on protein synthesis. Learn Mem 14: 497-503.

Jeyifous O, Waites CL, Specht CG, Fujisawa S, Schubert M, Lin EI, Marshall J, Aoki C, de Silva T, Montgomery JM, et al. 2009. SAP97 and CASK mediate sorting of NMDA receptors through a previously unknown secretory pathway. Nat Neurosci 12: 1011-1019.

Kogan JH, Frankland PW, Silva AJ. 2000. Long-term memory underlying hippocampus-dependent social recognition in mice. Hippocampus 10: $47-56$.

Kubota O, Hattori K, Hashimoto K, Yagi T, Sato T, Iyo M, Yuasa S. 2004 Auditory-conditioned-fear-dependent c-Fos expression is altered in the emotion-related brain structures of Fyn-deficient mice. Brain Res Mol Brain Res 130: 149-160.

Lau LF, Huganir RL. 1995. Differential tyrosine phosphorylation of $N$-methyl-D-aspartate receptor subunits. J Biol Chem 270: 20036-20041.

Lavezzari G, McCallum J, Dewey CM, Roche KW. 2004. Subunit-specific regulation of NMDA receptor endocytosis. J Neurosci 24: 6383-6391.

LeDoux JE. 2000. Emotion circuits in the brain. Annu Rev Neurosci 23: $155-184$.

Lee H, Kim JJ. 1998. Amygdalar NMDA receptors are critical for new fear learning in previously fear-conditioned rats. J Neurosci 18: 8444-8454.

Li XF, Phillips R, LeDoux JE. 1995. NMDA and non-NMDA receptors contribute to synaptic transmission between the medial geniculate body and the lateral nucleus of the amygdala. Exp Brain Res 105: $87-100$.

Maren S. 1999a. Long-term potentiation in the amygdala: A mechanism for emotional learning and memory. Trends Neurosci 22: 561-567.

Maren S. 1999b. Neurotoxic basolateral amygdala lesions impair learning and memory but not the performance of conditional fear in rats. J Neurosci 19: 8696-8703.

Maren S, Fanselow MS. 1995. Synaptic plasticity in the basolateral amygdala induced by hippocampal formation stimulation in vivo. J Neurosci 15: 7548-7564.

Maren S, Poremba A, Gabriel M. 1991. Basolateral amygdaloid multi-unit neuronal correlates of discriminative avoidance learning in rabbits. Brain Res 549: 311-316. 
Maren S, Yap SA, Goosens KA. 2001. The amygdala is essential for the development of neuronal plasticity in the medial geniculate nucleus during auditory fear conditioning in rats. J Neurosci 21: pRC135.

Markham CM, Huhman KL. 2008. Is the medial amygdala part of the neural circuit modulating conditioned defeat in Syrian hamsters? Learn Mem 15: 6-12.

McKernan MG, Shinnick-Gallagher P. 1997. Fear conditioning induces a lasting potentiation of synaptic currents in vitro. Nature 390: $607-611$.

Meredith M, Westberry JM. 2004. Distinctive responses in the medial amygdala to same-species and different-species pheromones. J Neurosci 24: $5719-5725$.

Monyer H, Burnashev N, Laurie DJ, Sakmann B, Seeburg PH. 1994. Developmental and regional expression in the rat brain and functional properties of four NMDA receptors. Neuron 12: 529-540.

Nakazawa T, Komai S, Tezuka T, Hisatsune C, Umemori H, Semba K, Mishina M, Manabe T, Yamamoto T. 2001. Characterization of Fyn-mediated tyrosine phosphorylation sites on GluRe2 (NR2B) subunit of the $N$-methyl-D-aspartate receptor. J Biol Chem 276: 693-699.

Nakazawa T, Tezuka T, Yamamoto T. 2002. Regulation of NMDA receptor function by Fyn-mediated tyrosine phosphorylation. Nihon Shinkei Seishin Yakurigaku Zasshi 22: 165-167.

Nakazawa T, Komai S, Watabe AM, Kiyama Y, Fukaya M, Arima-Yoshida F, Horai R, Sudo K, Ebine K, Delawary M, et al. 2006. NR2B tyrosine phosphorylation modulates fear learning as well as amygdaloid synaptic plasticity. EMBO J 25: $2867-2877$.

Petrulis A. 2009. Neural mechanisms of individual and sexual recognition in Syrian hamsters (Mesocricetus auratus). Behav Brain Res 200: 260-267.

Phelps EA, LeDoux JE. 2005. Contributions of the amygdala to emotion processing: From animal models to human behavior. Neuron 48: $175-187$.

Prybylowski K, Chang K, Sans N, Kan L, Vicini S, Wenthold RJ. 2005. The synaptic localization of NR2B-containing NMDA receptors is controlled by interactions with PDZ proteins and AP-2. Neuron 47: $845-857$.

Quirk GJ, Repa C, LeDoux JE. 1995. Fear conditioning enhances short-latency auditory responses of lateral amygdala neurons: Parallel recordings in the freely behaving rat. Neuron 15: 1029-1039.

Radley JJ, Farb CR, He Y, Janssen WG, Rodrigues SM, Johnson LR, Hof PR, LeDoux JE, Morrison JH. 2007. Distribution of NMDA and AMPA receptor subunits at thalamo-amygdaloid dendritic spines. Brain Res 1134: $87-94$.

Richter K, Wolf G, Engelmann M. 2005. Social recognition memory requires two stages of protein synthesis in mice. Learn Mem 12: 407-413.

Salter MW, Kalia LV. 2004. Src kinases: A hub for NMDA receptor regulation. Nat Rev Neurosci 5: 317-328.

Schimanski LA, Nguyen PV. 2005. Mouse models of impaired fear memory exhibit deficits in amygdalar LTP. Hippocampus 15: 502-517.

Sigurdsson T, Doyere V, Cain CK, LeDoux JE. 2007. Long-term potentiation in the amygdala: A cellular mechanism of fear learning and memory. Neuropharmacology 52: 215-227.

Snyder EM, Nong Y, Almeida CG, Paul S, Moran T, Choi EY, Nairn AC, Salter MW, Lombroso PJ, Gouras GK, et al. 2005. Regulation of NMDA receptor trafficking by amyloid- $\beta$. Nat Neurosci 8: 1051-1058.

Tovar KR, Westbrook GL. 2002. Mobile NMDA receptors at hippocampal synapses. Neuron 34: 255-264.

Yu XM, Askalan R, Keil GJ II, Salter MW. 1997. NMDA channel regulation by channel-associated protein tyrosine kinase Src. Science 275: $674-678$.

Zhang S, Edelmann L, Liu J, Crandall JE, Morabito MA. 2008. Cdk5 regulates the phosphorylation of tyrosine $1472 \mathrm{NR} 2 \mathrm{~B}$ and the surface expression of NMDA receptors. J Neurosci 28: 415-424.

Received January 29, 2010; accepted in revised form June 10, 2010. 


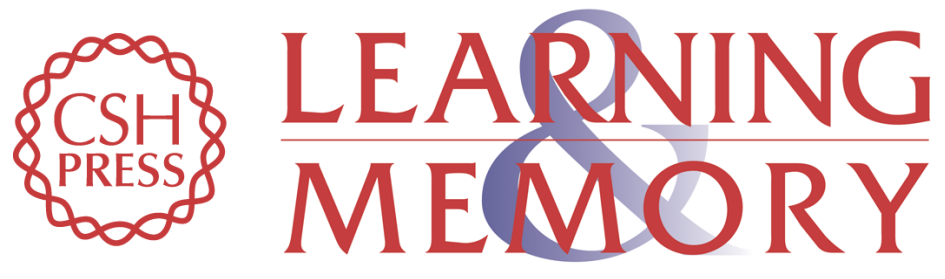

\section{Src inhibition reduces NR2B surface expression and synaptic plasticity in the amygdala}

Laleh Sinai, Steven Duffy and John C. Roder

Learn. Mem. 2010, 17:

Access the most recent version at doi:10.1101//m.1765710

References This article cites 45 articles, 16 of which can be accessed free at:

http://learnmem.cshlp.org/content/17/8/364.full.html\#ref-list-1

License

Email Alerting Receive free email alerts when new articles cite this article - sign up in the box at the Service top right corner of the article or click here. 